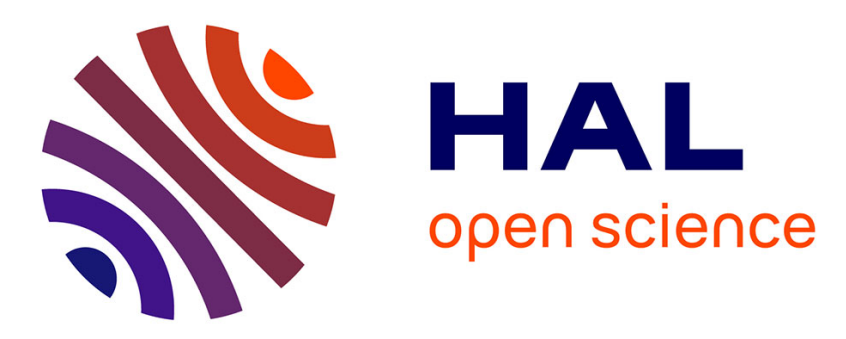

\title{
Photorefractive beam coupling in GaAs and InP generated by nanosecond light pulses
}

\author{
Jean Claude Fabre, Jean-Michel Jonathan, Gérald Roosen
}

\section{To cite this version:}

Jean Claude Fabre, Jean-Michel Jonathan, Gérald Roosen. Photorefractive beam coupling in GaAs and InP generated by nanosecond light pulses. Journal of the Optical Society of America B, 1988, 5

(8), pp.1730-1736. hal-00862107

\section{HAL Id: hal-00862107 \\ https://hal-iogs.archives-ouvertes.fr/hal-00862107}

Submitted on 16 Sep 2013

HAL is a multi-disciplinary open access archive for the deposit and dissemination of scientific research documents, whether they are published or not. The documents may come from teaching and research institutions in France or abroad, or from public or private research centers.
L'archive ouverte pluridisciplinaire HAL, est destinée au dépôt et à la diffusion de documents scientifiques de niveau recherche, publiés ou non, émanant des établissements d'enseignement et de recherche français ou étrangers, des laboratoires publics ou privés. 


\title{
Photorefractive beam coupling in GaAs and InP generated by nanosecond light pulses
}

\author{
J. C. Fabre, J. M. C. Jonathan, and G. Roosen \\ Institut d'Optique, Bâtiment 503, Unité Associée au Centre National de la Recherche Scientifique, Centre \\ Universitaire, B.P. 43, 91406 Orsay Cedex, France
}

Received January 4, 1988; accepted March 28, 1988

\begin{abstract}
Energy transfer between two nanosecond light pulses interacting in semi-insulating $\overline{4} 3 \mathrm{~m}$ crystals is studied. By using the specific dependences of the photorefractive effect on wave polarizations and crystal orientation, the contribution of this to two-wave mixing in GaAs and InP is analyzed.
\end{abstract}

\section{INTRODUCTION}

Coherent optics offers unique features for information processing and transmission with a great parallelism and a high data rate. However, the implementation of these ideas for valuable processor demonstration requires nonlinear-optical materials that permit efficient interactions between optical beams.

Electro-optic and photoconductive materials are of considerable interest as they allow for large nonlinearities with low-power laser beams through the photorefractive effect. This effect has also proven to be fast when high-intensity light pulses are used for excitation. ${ }^{1-7}$

Using photorefractive materials such as $\mathrm{Bi}_{12} \mathrm{SiO}_{20}$ (BSO), $\mathrm{Bi}_{12} \mathrm{GeO}_{20}$ (BGO), $\mathrm{BaTiO}_{3}$, and $\mathrm{KNbO}_{3}$, a wide variety of operations has been demonstrated in fields such as dynamic holography, ${ }^{8}$ optical memories, ${ }^{9,10}$ optical phase conjugation, ${ }^{11}$ spatial light modulators, ${ }^{12}$ or optical interconnections. ${ }^{13}$

A new field of interest has been discovered recently from evidence that the photorefractive effect can be induced in the near infrared in compound semiconductors such as GaAs or InP. Four-wave mixing and energy transfer in two-beamcoupling experiments have been demonstrated in such materials. ${ }^{5,14-19}$

Photorefractive semiconductor crystals are of great interest as they promise efficient optical processing in the near infrared. High-quality crystals are available, and their optical and electronic properties are adjustable according to the envisaged applications. The optical energy required to generate the photorefractive effect in these materials is low enough to permit the use of laser diodes. Moreover, because of the high carrier mobility, it should be possible to reach response times in the picosecond or even subpicosecond résponse times.

We present here results on energy transfer between two nanosecond light pulses at $1.06 \mu \mathrm{m}$, interacting in semiinsulating $\mathrm{GaAs}$ and $\mathrm{InP}$ crystals. The experiments confirm the fast photorefractive effect in both crystals. However, the effect has to be separated from other nonlinear phenomena, such as light-induced absorption. This can be done by using the characteristic symmetries of the electro-optic tensor. We will devote special attention to those symmetries and will show that efficient energy transfer can be obtained even in nonstandard configurations.

\section{EXPERIMENTAL TECHNIQUES}

Two-beam-mixing energy-transfer experiments were performed in two $n$-type semi-insulating crystals: a chromiumdoped GaAs and an iron-doped InP.

The properties of our samples are inferred from measurements and from the literature, as given in Tables 1 and 2 .

The laser source is a Nd:YAG laser emitting 3-nsec pulses, with energies ranging from 0.5 to $6 \mathrm{~mJ} / \mathrm{cm}^{2}$. The setup is shown in Fig. 1. Because of the circular polarization of the output beam, a quarter-wave plate $(\lambda / 4)$ and a crystal polarizer are used to achieve linear polarizations perpendicular to the figure plane. This also allows us to adjust the beam energy. Using beam splitter BS1, a pump beam and a weaker probe beam ( $1 \%$ of pump) are obtained. They interfere in the sample, generating an illumination grating of a period of $1 \mu \mathrm{m}$. Half-wave plates $(\lambda / 2)$ on each beam permit the rotation of the beam polarizations.

After transmission through the crystal, the probe beam is detected by a fast silicon photodiode (PD2). A second one (PD1) detects a fraction of the same beam, extracted before the crystal by beam splitter BS2. The reference signal from PD1, electronically delayed, is mixed with that from PD2 at the input of a fast oscilloscope. The delay is adjusted for convenient observation of the two signals on a single oscilloscope trace. With no pump beam incident upon the crystal, the two signals are made equal by using a neutral optical density filter in the reference branch [Fig. 2(a)]. With both the probe and the pump beams illuminating the crystal, a gain [Fig. 2(b)] or a depletion [Fig. 2(c)] in the energy of the probe beam is detected. The relative amplification or gain of the probe beam is defined as

$$
\gamma=\frac{I_{\text {probe }}(\text { with pump })}{I_{\text {probe }}(\text { without pump })}=\exp (\Gamma l),
$$


Table 1. Parameters of GaAs Crystal

\begin{tabular}{|c|c|c|}
\hline Parameter & Value & Ref. \\
\hline$n$ (refractive index) & 3.48 & 20 \\
\hline$\epsilon_{r}$ (relative dielectric constant) & 13 & 20 \\
\hline$r_{41}$ (electro-optic coefficient) $\left(\mathrm{pm} \mathrm{V}^{-1}\right)$ & 1.35 & 21 \\
\hline [Cr] (chromium density) $\left(\mathrm{cm}^{-3}\right)$ & $10^{17}$ & 21 \\
\hline$\alpha$ (absorption coefficient) $\left(\mathrm{cm}^{-1}\right)$ & $1.5 \pm 0.2$ & \\
\hline$\sigma_{0}$ (dark conductivity) $\left(\Omega^{-1} \mathrm{~cm}^{-1}\right)$ & $10^{-8}$ & \\
\hline Crystal size along $[110](\mathrm{cm})$ & 0.5 & \\
\hline Crystal size along [001] $(\mathrm{cm})$ & 0.55 & \\
\hline Crystal size along $[\overline{1} 10]^{a}(\mathrm{~cm})$ & 0.4 & \\
\hline
\end{tabular}

${ }^{a}$ Direction of light propagation.

\section{Table 2. Parameters of InP Crystal}

\begin{tabular}{lcc}
\hline \multicolumn{1}{c}{ Parameter } & Value & Ref. \\
\hline$n$ (refractive index) & 3.3 & 14 \\
$\epsilon_{r}$ (relative dielectric constant) & 12.7 & 14 \\
$r_{41}$ (electro-optic coefficient) $\left(\mathrm{pm} \mathrm{V}^{-1}\right)$ & 1.45 & 14 \\
[Fe] (iron density) $\left(\mathrm{cm}^{-3}\right)$ & $10^{17}$ & 22 \\
$\alpha$ (absorption coefficient) $\left(\mathrm{cm}^{-1}\right)$ & $1.4 \pm 0.2$ & \\
$\sigma{\text { (photoconductivity) }\left(\Omega^{-1} \mathrm{~cm}^{-1}\right)}^{\left(\text {with } I \text { in W } \mathrm{cm}^{-2}\right)}$ & $(1.3+3 I) 10^{-8}$ & \\
Crystal size along [110] $(\mathrm{cm})$ & & \\
Crystal size along [001] $(\mathrm{cm})$ & 0.75 & \\
Crystal size along [110] ${ }^{a}(\mathrm{~cm})$ & 0.45 & \\
\hline
\end{tabular}

${ }^{a}$ Direction of light propagation.

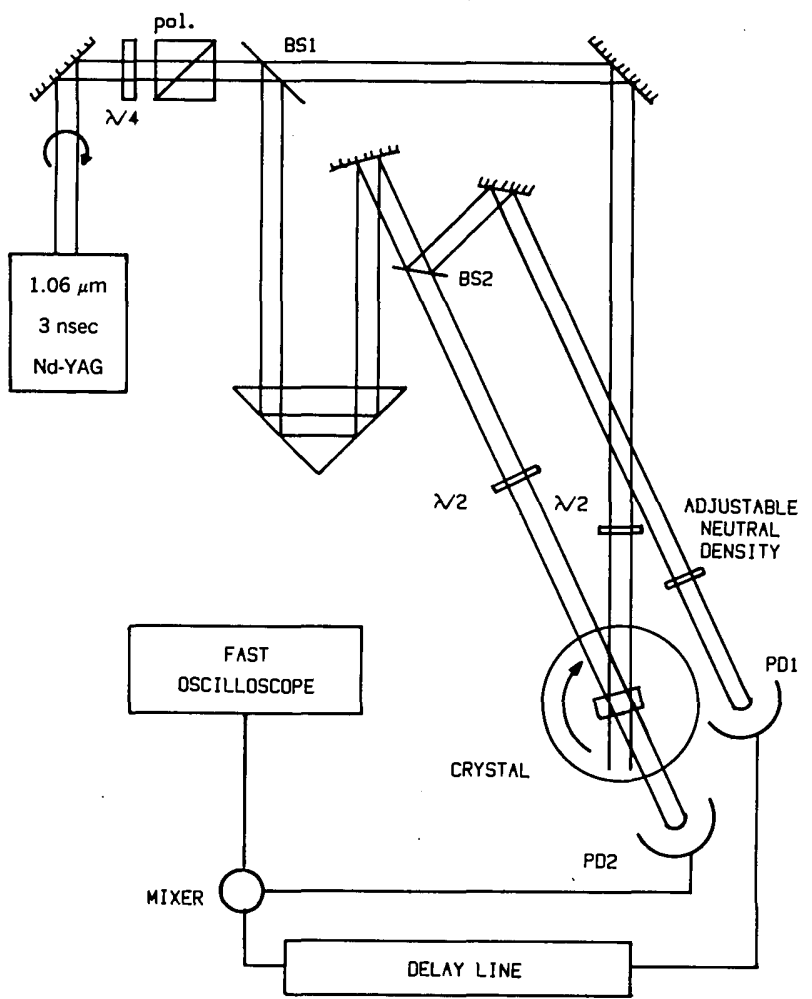

Fig. 1. Experimental setup for energy-transfer measurements in . semiconductors at $1.06 \mu \mathrm{m}$. where $\Gamma$ is the coupling gain per unit length and $l$ is the interaction length. $\gamma$ is measured by the ratio of the signals from the two detectors. This method permits consistent measurements of the coupling gain $(\Gamma l)$ despite strong fluctuations in the pulse energy (15-20\%). The final value for $\gamma$ is taken as the mean value from several experiments.

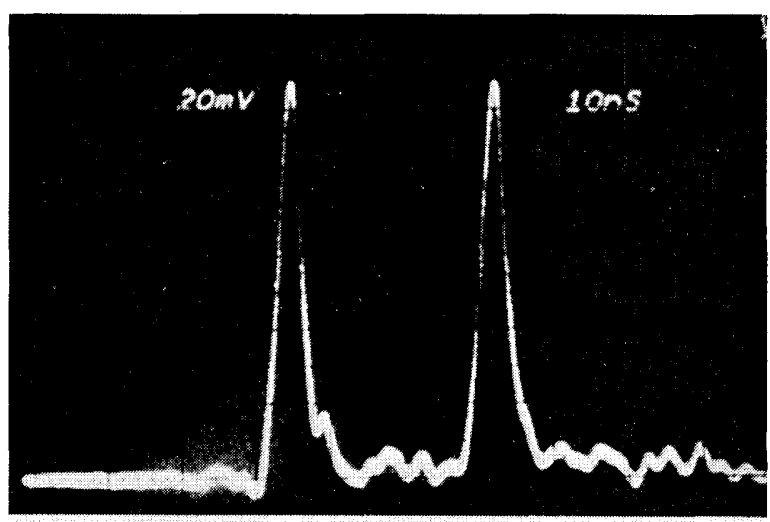

(a)

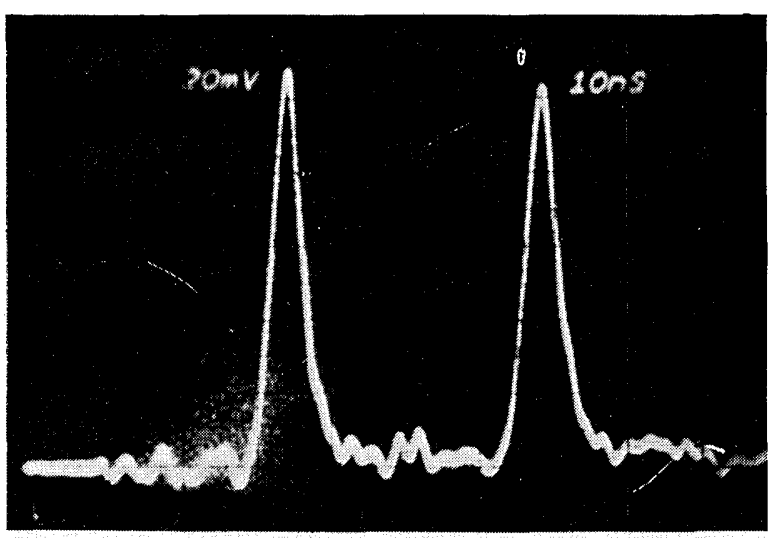

(b)

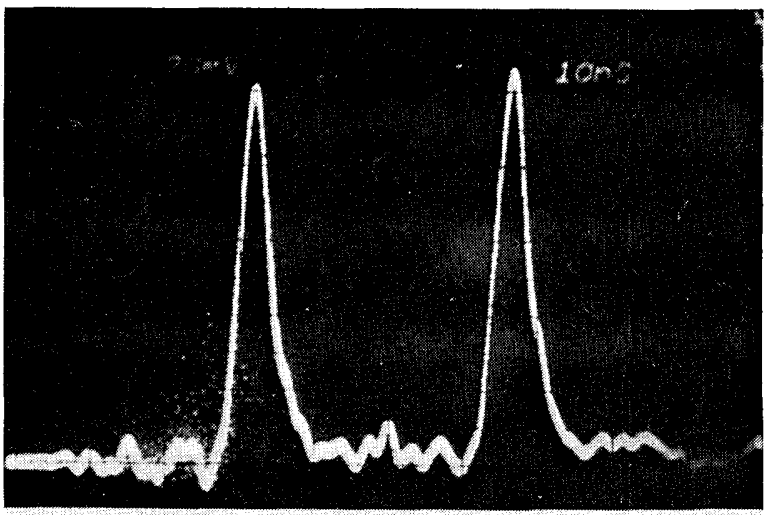

(c)

Fig. 2. Oscilloscope traces of the transmitted probe pulse (PD2) followed by delayed reference pulse (PD1): (a) equalization of the signal, without pump beam, (b) depletion of the probe beam, with pump beam, (c) amplification of the probe beam, with pump beam. The vertical scale is $20 \mathrm{mV}$ per division, and the horizontal scale is $10 \mathrm{nsec}$ per division. 


\section{SYMMETRIES OF THE PHOTOREFRACTIVE ENERGY TRANSFER}

In the photorefractive effect the induced variations of the relative dielectric tensor $\epsilon_{r}$ are given in terms of the electrooptic tensor $\mathbf{R}$ and of the space-charge field $\mathbf{E}_{1}$ by ${ }^{11}$

$$
\left(\Delta \boldsymbol{\epsilon}_{\mathbf{r}}\right)=-\epsilon_{\mathbf{r}}(\mathbf{R} \cdot \hat{k}) \boldsymbol{\epsilon}_{\mathbf{r}} \cdot E_{1},
$$

where $\hat{k}$ is the unit vector parallel to the grating vector $\mathbf{k}$ and $E_{1}$ is the complex amplitude of the space-charge field.11 In photorefractive crystals in the diffusion regime (no applied electric field) the phase shift between the index grating and the illumination grating is $\pm \pi / 2$, depending on the sign of the photocarriers and the orientation of the crystal. Thus $E_{1}= \pm m E_{\text {sc }}$, where $m$ is the modulation ratio of the interference pattern $(m \ll 1)$ and $E_{\mathrm{sc}}=E_{d} E_{q} /\left(E_{d}+E_{q}\right), E_{d}$ and $E_{q}$ being the diffusion and limiting fields, respectively, as defined in Ref. 23.

The photorefractive coupling gain per unit length of interaction of the two optical waves $\xi_{1}$ (probe) and $\xi_{2}$ (pump) is then

$$
\Gamma= \pm \frac{2 \pi}{\lambda} \frac{r_{\text {eff }}}{n} E_{\mathrm{sc}} \frac{1}{\cos \theta}\left(\hat{e}_{1} \cdot \hat{e}_{2}^{*}\right)
$$

where $\theta$ is the half-angle between the two beams inside the crystal and $n$ is the refractive index at wavelength $\lambda$. The effective electro-optic coefficient $r_{\text {eff }}$ is given by

$$
r_{\text {eff }}=\hat{e}_{2}{ }^{*} \epsilon_{\mathbf{r}}(\mathbf{R} \cdot \hat{k}) \epsilon_{\mathbf{r}} \hat{e}_{1}
$$

and thus depends on the orientation of the crystal and on the polarizations $\hat{e}_{1}$ and $\hat{e}_{2}$ of the two waves. ${ }^{17,19}$

According to the symmetries of the (23) and ( $\overline{4} 3 \mathrm{~m})$ crystals, the electro-optic tensor $\mathbf{R}$ may be written as

$$
\begin{aligned}
\mathbf{R}= & r_{41}(\hat{x} \otimes \hat{y} \otimes \hat{z}+\hat{x} \otimes \hat{z} \otimes \hat{y}+\hat{y} \otimes \hat{x} \otimes \hat{z}+\hat{y} \otimes \hat{z} \otimes \hat{x} \\
& +\hat{z} \otimes \hat{x} \otimes \hat{y}+\hat{z} \otimes \hat{y} \otimes \hat{x}),
\end{aligned}
$$

where $\hat{x}, \hat{y}$, and $\hat{z}$ are the unit vectors of the crystallographic axis and $\otimes$ denotes the tensorial product of two vectors. Using the relation

$$
(\hat{i} \otimes \hat{j} \otimes \hat{k}) \cdot \mathbf{V}=(\hat{i} \otimes \hat{j})(\mathbf{k} \cdot \mathbf{V}),
$$

we may easily calculate $r_{\text {eff. }}$

\section{Beam-Coupling Configuration}

In cubic crystals such as optically active BSO or BGO (23 symmetry group) and isotropic GaAs or InP ( $\overline{4} 3 \mathrm{~m}$ symmetry group), energy-transfer experiments are mostly carried out with the grating vector $\mathbf{k}$ parallel to the (001) axis. That is, the so-called beam-coupling configuration (Fig. 3). In such a situation, $\hat{k}=\hat{z}$, and the central term $(\mathbf{R} \cdot \hat{k})$ in Eq. (4) reduces to

$$
(\mathbf{R} \cdot \hat{k})=r_{41}(\hat{x} \otimes \hat{y}+\hat{y} \otimes \hat{x}),
$$

and the polarizations $\hat{e}_{1}$ and $\hat{e}_{2}$ of the two optical beams are assumed to be eigenpolarizations of the crystal, i.e., any polarization for the $\overline{4} 3 \mathrm{~m}$ crystal.

If $\theta$ is half the angle between these two beams inside the crystal and $\alpha$ is that of both electric fields relative to the [110] axis (Fig. 3), $r_{\text {eff }}$ may be written as

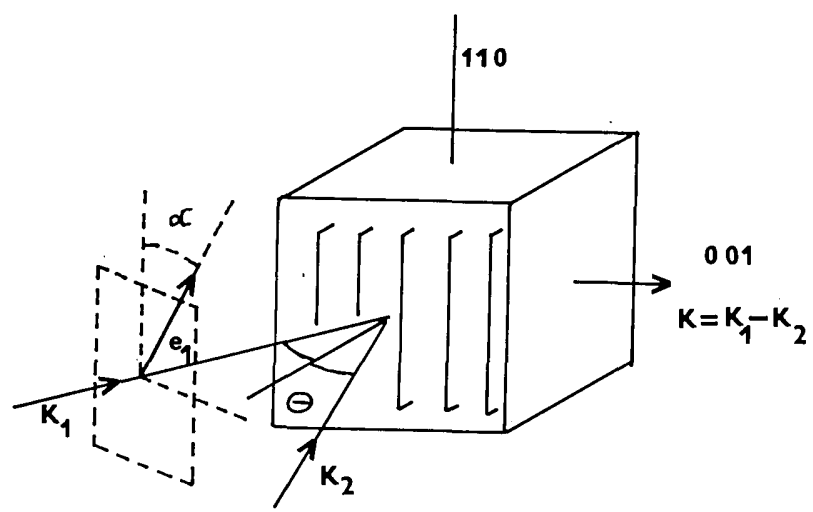

Fig. 3. The so-called beam-coupling configuration.

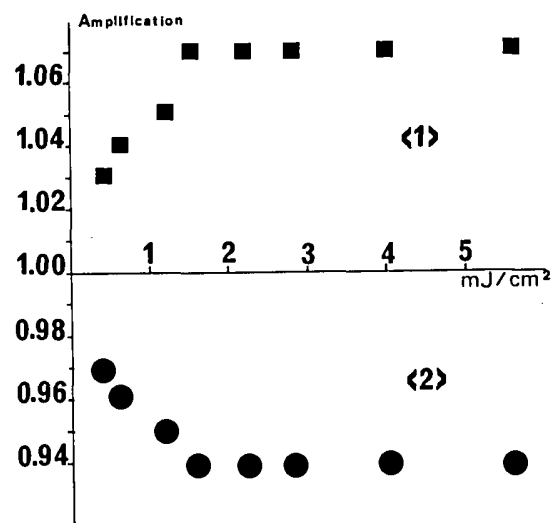

Fig. 4. Beam coupling in GaAs in the coupling configuration. Rotation of $180^{\circ}$ of the crystal about the [110] axis (orientations $\langle 1\rangle$ and $\langle 2\rangle)$ reverses the energy-transfer direction.

$$
r_{\text {eff }}=r_{41} n^{4}\left(\sin ^{2} \alpha \sin ^{2} \theta+\cos ^{2} \alpha\right) .
$$

The effective electro-optic coefficient is thus maximum when both waves are linearly polarized along [110] (i.e., perpendicular to $\hat{k}: \alpha=0$ ). Equation (8) then shows no dependence on the grating spatial frequency.

A straightforward calculation gives the well-known result that rotation of the crystal through $180^{\circ}$ around [110] changes the sign of $r_{\text {eff }}$, therefore also changing that of the energy transfer. This symmetry is important in order to characterize the photorefractive origin of the energy-transfer process. Such a behavior is illustrated by Fig. 4, in which the probe-beam gain amplification $\left(\gamma_{1}\right)$ or depletion $\left(\gamma_{2}\right)$, depending on the crystal orientation, is plotted as functions of the pulse energy for a GaAs sample.

One finds that $\gamma_{1}+\gamma_{2} \simeq 0$, which implies that the observed energy transfer is only due to the photorefractive effect.

\section{Diffraction Configuration}

In the diffraction-efficiency configuration the wave vectors of the two interfering waves (therefore the grating vector itself) are perpendicular to the [001] axis (Fig. 5). This configuration is extensively used for the photorefractive characterization of optically active crystals belonging to the (23) symmetry group, ${ }^{24}$ such as BSO and BGO. In that case, from Eq. (3) and using circular polarizations that are the eigenpolarizations of optically active materials, one easily shows that $r_{\text {eff }}$ is zero unless $\hat{e}_{1}$ and $\hat{e}_{2}$ are orthogonal eigenpo- 
larizations. Thus there is a possibility of energy transfer by diffraction of a circularly polarized wave into the orthogonal one, but no real beam coupling occurs between interfering waves with the same eigenpolarization. For this reason, beam-coupling experiments were not' performed in this configuration.

However, it is of interest in isotropic $\overline{4} 3 \mathrm{~m}$ crystals. In that case the unit grating vector $\hat{k}$ is

$$
\hat{k}=\frac{1}{\sqrt{2}}(\hat{x}+\hat{y})
$$

and $(\mathbf{R} \cdot \hat{k})$, the central term in Eq. (4), may be written as

$$
(\mathbf{R} \cdot \hat{k})=\frac{r_{41}}{\sqrt{2}}(\hat{y} \otimes \hat{z}+\hat{z} \otimes \hat{y}+\hat{x} \otimes \hat{z}+\hat{z} \otimes \hat{x}) .
$$

When the same definitions as before are used for $\theta$ and $\alpha$ (Fig. 5), the effective electro-optic coefficient becomes

$$
r_{\text {eff }}=r_{41} n^{4} \sin (2 \alpha) \cos \theta .
$$

It is zero for an incident rectilinear polarization parallel to the [001] axis. Again there is a possibility for energy transfer by diffraction of a linear polarized wave into the orthogonal one, but coherent coupling between interfering beams is forbidden.

However, for an incident polarization at $45^{\circ}$ from the [001] axis, the effective electro-optic coefficient is a maximum. For that orientation and small values of $\theta$ (i.e., large grating

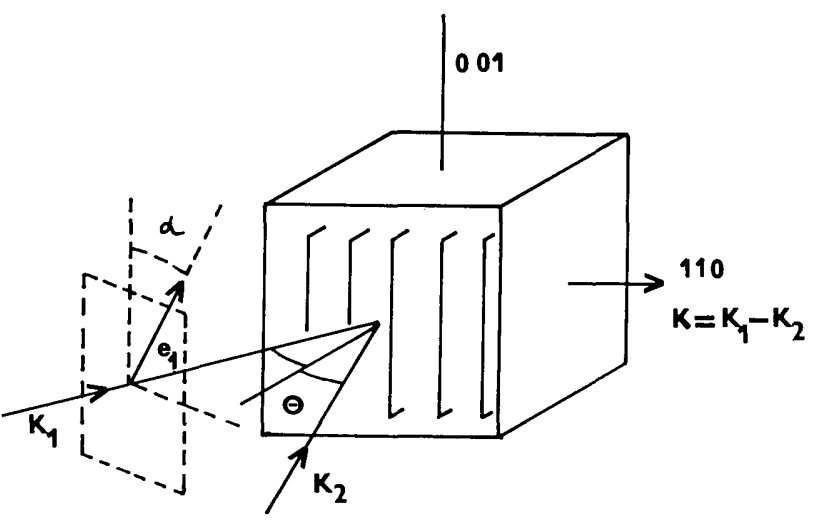

Fig. 5. The so-called diffraction-efficiency configuration.

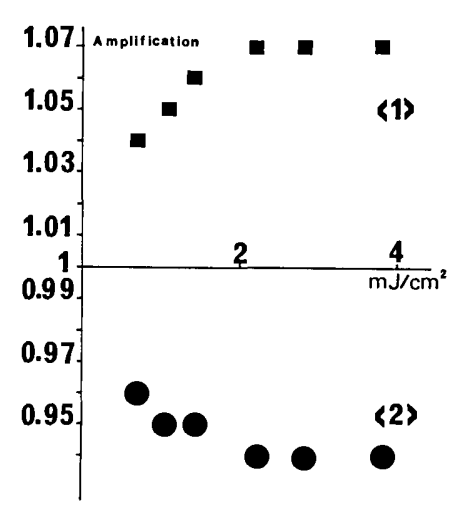

Fig. 6. Beam coupling in GaAs in the diffraction configuration. A $90^{\circ}$ rotation of the beam polarizations (polarizations $\langle 1\rangle$ and $\langle 2\rangle$ ) reverses the energy-transfer direction. periods), $r_{\text {eff }}$ has the same value in this so-called diffraction configuration as in the beam-coupling configuration, and efficiency of the energy transfer is similar. An interesting feature is the polarization dependence of $r_{\text {eff: }}$ a $90^{\circ}$ rotation of the polarizations of the interfering beams will reverse the energy transfer, as did a rotation of the crystal in the coupling configuration. This is illustrated by Fig. 6. The relative gain $\gamma$ of the probe beam versus pulse energy in the diffraction configuration has been plotted for the two polarizations. The symmetry is observed, and values for this relative amplification fit those obtained in the coupling configuration.

Let us point out the fact that in this configuration a $180^{\circ}$ rotation of the crystal does not necessarily lead to a reversal of the energy transfer. A straightforward calculation of $r_{\text {eff }}$ shows that there is no reversal through $180^{\circ}$ rotation around the [001] axis. Reversal appears if the input and output faces of the crystal are exchanged through a $180^{\circ}$ rotation around the [110] axis. This may be understood by the fact that the polarizations are changed and the $\hat{k}$ vector is inverted in the first case, each one of those two modifications leading to a change in the sign of $r_{\text {eff }}$. Only the polarizations are changed in the second case. A third possibility would be a rotation around the axis of the input and output faces that maintains the polarizations but reverses the $k$ vector. In that case too, the sign of the energy transfer is changed.

\section{EXPERIMENTAL RESULTS AND DISCUSSION}

The energy transfer in GaAs has been studied as a function of the energy of the pump beam. Results are illustrated in Figs. 4 and 6, which show reasonably symmetrical amplifications according to the predicted symmetries.

Similar experiments have been made using an iron-doped InP crystal. Figure 7 gives a plot of the relative amplifications $\gamma_{1}$ and $\gamma_{2}$ in the beam-coupling configuration for the two positions of the crystal that interchange the input and output faces. A strong nonsymmetrical behavior is observed, pointing out the influence of a nonphotorefractive effect.

In the small-gain approximation this behavior can be depicted by considering the relative gain of the probe beam $\left[\gamma_{1,2}=\exp \left(\Gamma_{1,2} l\right)\right]$ as the sum of two terms. The symmetrical term, $\gamma_{\mathrm{pr}}$, corresponds to the photorefractive effect; it can be extracted from the two measurements of the relative gain by

$$
\gamma_{\mathrm{pr}}=1 / 2\left(\gamma_{1}-\gamma_{2}\right)
$$

The nonsymmetrical contribution to the gain, $\gamma_{\mathrm{npr}}$, corresponds to a nonphotorefractive phenomenon; it is given by

$$
\gamma_{\mathrm{npr}}=1-1 / 2\left(\gamma_{1}+\gamma_{2}\right)
$$

Results obtained in the GaAs and InP crystals are given in Figs. 8 and 9, respectively.

As anticipated from the results in Figs. 4 and 6, the only contribution to energy transfer in GaAs comes from the photorefractive effect (Fig. 8).

InP (Fig. 9) exhibits a photorefractive effect of the same order of magnitude as GaAs. However, there is an important nonphotorefractive contribution to the relative gain that is superimposed onto the photorefractive one. This produces an apparent increase of the crystal absorption in 


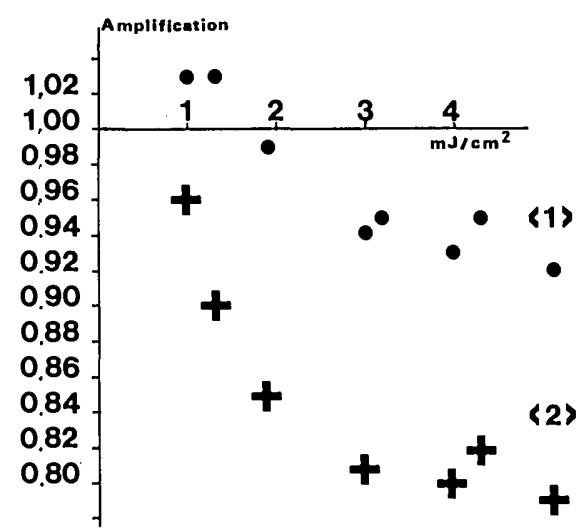

Fig. 7. Energy transfer in InP in the coupling configuration: change in the amplification direction through $180^{\circ}$ rotation of the crystal around its [110] axis (orientations $\langle 1\rangle$ and $\langle 2\rangle$ ).

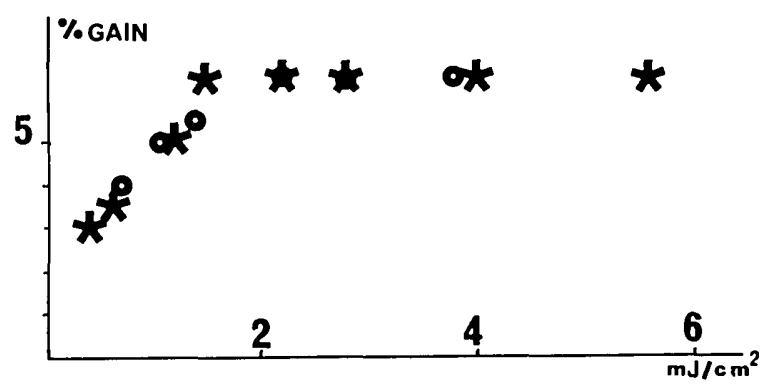

Fig. 8. Relative amplification of the probe beam owing to the photorefractive effect in GaAs: *, beam-coupling configuration; $O$, diffraction configuration.

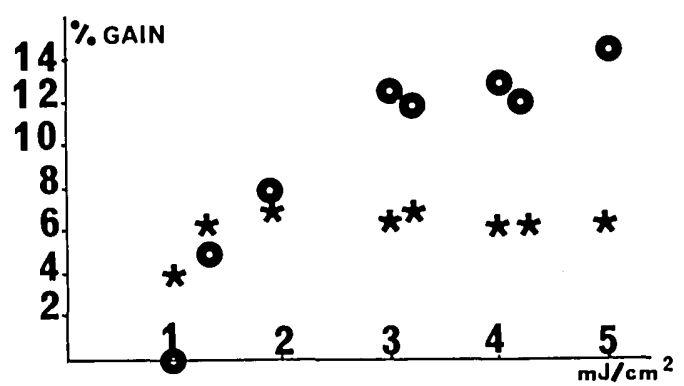

Fig. 9. Relative amplification in InP, which is attributed to the *, photorefractive effect by symmetrization and $O$, contribution of the induced absorption.

the presence of the pump beam. This effect is not due to any population grating, as the same induced variation in the absorption coefficient arises for crossed polarizations of pump and probe beams (Fig. 10). The experiments were made by measuring the relative amplification $\gamma_{1}$ and $\gamma_{2}$ for the two orientations of the crystal. Results are reported in Fig. 11 and exhibit accurate coincidence with the points extracted from previous measurements by using Eq. (9). The physical origin of this phenomenon in these experiments is not fully understood yet.

From the literature (see, for example, Refs. 25-27) it appears that the generation of charge carriers resulting from optical excitation at $\lambda=1.06 \mu \mathrm{m}$ could involve various optical and thermal transitions. Further investigations are needed to determine their relative contributions in our ex- periments and to reach quantitative agreement between theory and experimental results. We will devote special attention to the relaxation kinetics of the induced absorption and wavelength dependence.

From the results just described, one can draw the following conclusions: In the short pulse regime (pulse duration $T_{L}$ much less than recombination $\tau_{R}$ and diffusion $\tau_{D}$ times) the space-charge field develops through diffusion or drift of the photoexcited carriers after the illumination end. $5,7,28$ Consequently it cannot be used for two-wave-mixing experiments.

Consider now the opposite case, i.e., the quasi-cw regime. It is assumed that the unmodulated part of the photoexcited charge density reaches its steady-state value before drift or diffusion occurs. The space charge builds up from repeated excitation, diffusion, or drift and recombination of the carriers.

Observing a photorefractive energy transfer between light pulses indicates that a space-charge electrostatic field is built in 3 nsec. Indeed, at low gains the time evolutions of the photorefractive gain and space-charge field are identical; therefore we probably observe either a quasi-cw regime or an intermediate one.

Keeping in mind the fact that the magnitude of the photorefractive effect depends on energy, we can set forth two reasons to explain the invariance of the coupling gain with energy greater than $1.5 \mathrm{~mJ} \mathrm{~cm}^{-2}$. First, one could assume a

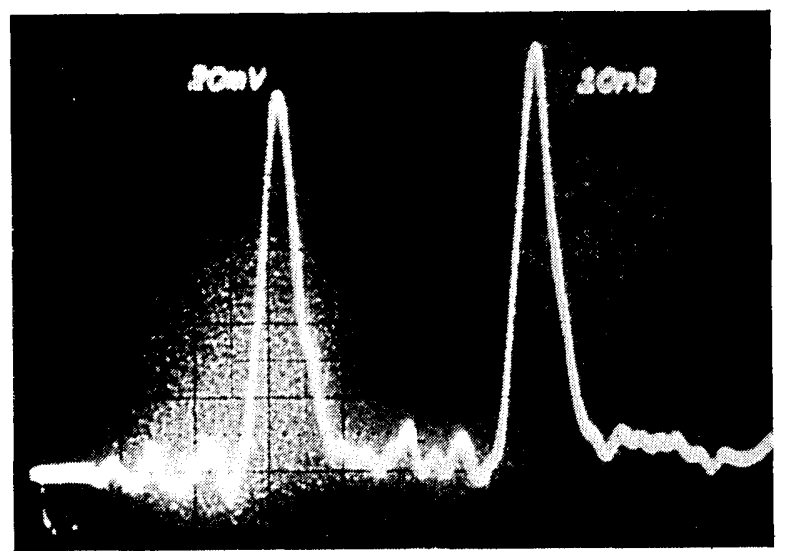

Fig. 10. Oscilloscope trace of the transmitted probe pulse followed by a delayed reference pulse. The polarizations of pump and probe pulses are orthogonal.

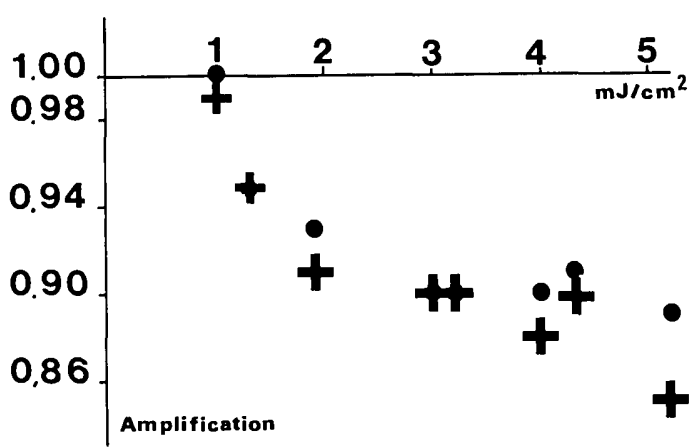

Fig. 11. Separate measurement of the induced absorption using orthogonally polarized waves. No noticeable influence of the crystal orientation is observed: $\bullet$, position $\langle 1\rangle ;+$, position $\langle 2\rangle$. 


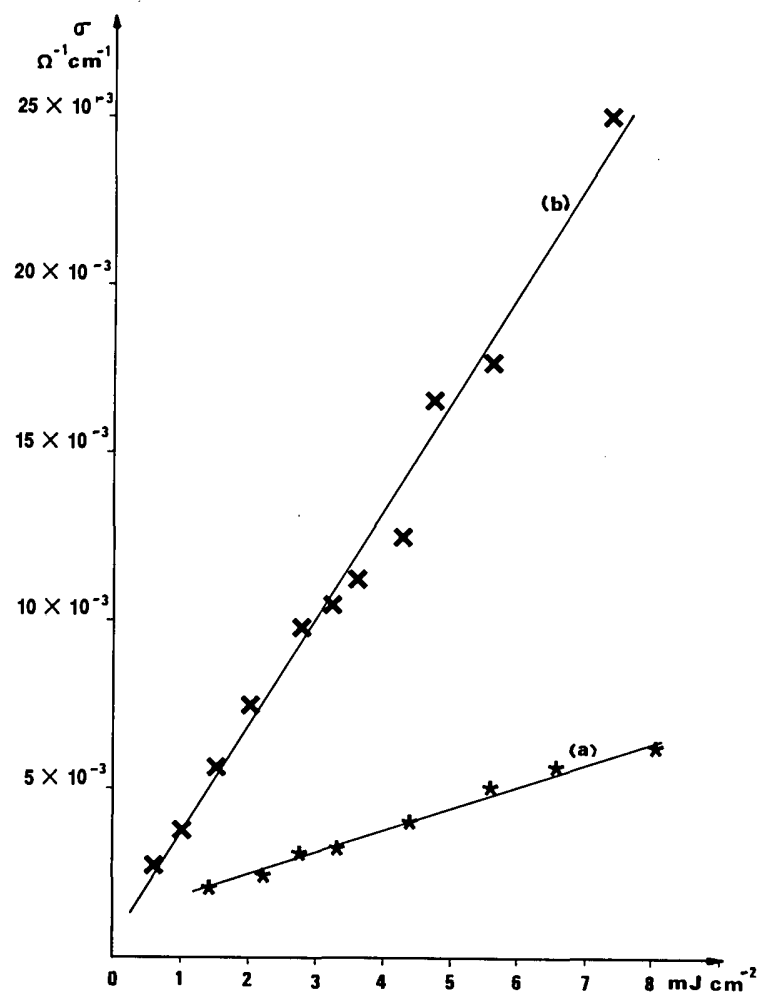

Fig. 12. Photoconductivity versus absorbed optical energy in curve (a) GaAs and curve (b) InP (applied voltage $V=180 \mathrm{~V}$ ).

limitation in the donor density. In fact, this assumption is denied by the linear variation of the photocurrent versus fluences indicated in this range (Fig. 12).

Second, an energy-independent coupling gain is well predicted for a photorefractive effect generated in the regime of quasi-continuous illumination. In this regime the illumination and the grating buildup times are much longer than both diffusion and recombination times of the excited charge carriers. The space-charge field builds up with a time constant $\tau_{e}$, which becomes faster with increasing light intensity. However, the magnitude of state level is independent of the light intensity.

Thus, in GaAs and InP, for fluences greater than $1.5 \mathrm{~mJ}$ $\mathrm{cm}^{-2}$, the steady-state photorefractive effect is reached within the pulse duration of 3 nsec. The observed steadystate value of $6.5 \%$ beam coupling corresponds to a gain per unit length $\Gamma=0.16 \mathrm{~cm}^{-1}$ for GaAs and $\Gamma=0.12 \mathrm{~cm}^{-1}$ for InP. This corresponds to a photoinduced space-charge field of $0.47 \mathrm{kV} / \mathrm{cm}$ for GaAs and $0.40 \mathrm{kV} / \mathrm{cm}$ for InP [Eq. (3)]. The effective density of trap sites derived from the quasi-cw model ${ }^{23}$ is then $3 \times 10^{14} \mathrm{~cm}^{-3}$ for GaAs and $2 \times 10^{14} \mathrm{~cm}^{-3}$ for InP. These values are slightly lower than previously published. ${ }^{15,16}$

At lower fluences, the power of the 3-nsec light pulse is not high enough to permit a complete buildup to the steadystate level in a time $\tau_{e}$ less than the pulse duration.

\section{CONCLUSION}

Quasi-steady-state behavior of the photorefractive effect in $\mathrm{GaAs}$ and InP is observed in the nanosecond regime for energy densities as low as $1.5 \mathrm{~mJ} \mathrm{~cm}^{-2}$. In the case of $\operatorname{InP}$, a strong induced absorption is separated from the photorefractive effect by using the symmetries of the electro-optic tensor.

Experiments are in progress to study both the intensitydependent absorption in InP and the photorefractive effect in both crystals through four-wave-mixing experiments.

\section{ACKNOWLEDGMENTS}

J. P. Herriau and J. P. Huignard (Thomson-CSF) provided the GaAs sample; B. Lambert and M. Coquille (CNET Lannion) provided the InP sample. We wish to thank them for that and for their interest in our work. We are also grateful to G. Colas and R. Leclerq (Institut d'Optique) for polishing the samples.

\section{REFERENCES}

1. C. T. Chen, D. M. Kim, and D. Von der Linde, "Efficient pulsed photorefractive process in $\mathrm{LiNbO}_{3}: \mathrm{Fe}$ for optical storage and deflection," IEEE J. Quantum Electron. QE-16, 126 (1980).

2. L. K. Kam, T. Y. Chang, J. Feinberg, and R. W. Hellwarth, "Photorefractive-index gratings formed by nanosecond optical pulses in $\mathrm{BaTiO}_{3}$," Opt. Lett. 6, 475 (1981).

3. J. P. Hermann, J. P. Herriau, and J. P. Huignard, "Nanosecond four wave mixing and holography in BSO crystals," Appl. Opt. 20, 2173 (1981).

4. G. Le Saux, G. Roosen, and A. Brun, "Observation and analysis of the fast photorefractive process in BSO," Opt. Commun. 56, 374 (1986); "Nanosecond light energy transfer in $\mathrm{Bi}_{12} \mathrm{SiO}_{20}$ at 532 nm," Opt. Commun. 58, 238 (1986).

5. G. C. Valley, A. L. Smirl, M. B. Klein, K. Bohnert, and T. F. Boggess, "Picosecond photorefractive beam coupling in GaAs," Opt. Lett. 11, 647 (1986).

6. A. L. Smirl, G. C. Valley, R. A. Mullen, K. Bohnert, C. D. Mire, and T. F. Boggess, "Picosecond photorefractive effect in $\mathrm{BaTiO}_{3}$," Opt. Lett. 12, 501 (1987).

7. J. M. C. Jonathan, Ph. Roussignol, and G. Roosen, "Time resolved buildup of a photorefractive grating induced in $\mathrm{Bi}_{12} \mathrm{SiO}_{20}$ by picoseond light pulses," Opt. Lett. 13, 224 (1988).

8. J. P. Huignard and F. Micheron, "High sensitivity read-write volume holographic storage in $\mathrm{Bi}_{12} \mathrm{SiO}_{20}$ and $\mathrm{Bi}_{12} \mathrm{GeO}_{20}$ crystals," Appl. Phys. Lett. 29, 591 (1976).

9. J. J. Amodei, "Electron diffusion effects during holographic recording in crystals," Appl. Phys. Lett. 18, 22 (1971).

10. A. M. Glass, G. E. Peterson, and T. J. Negran, in Laser Induced Damage in Optical Materials, A. J. Glass and A. H. Guenther, eds. (National Bureau of Standards, Washington D.C., 1976), pp. 1479-1484.

11. J. Feinberg, D. Heiman, A. R. Tanguay, and R. W. Hellwarth, "Photorefractive effects and light-induced charge migration in barium titanate," J. Appl. Phys. 51, 1297 (1980).

12. A. Marrakchi, A. R. Tanguay, J. Yu, and D. Psaltis, "Physical characterization of the photorefractive incoherent to coherent optical converter," Opt. Eng. 24, 124 (1985).

13. G. Pauliat, J. P. Herriau, A. Delboulbé, G. Roosen, and J. P. Huignard, "Dynamic beam deflection using photorefractive gratings in $\mathrm{Bi}_{12} \mathrm{SiO}_{20}$ crystals," J. Opt. Soc. Am. B 3, 306 (1986).

14. A. M. Glass, A. M. Johnson, D. H. Olson, W. Simpson, and A. A. Ballman, "Four wave mixing in semi-insulating InP and GaAs using the photorefractive effect," Appl. Lett. 44, 948 (1984).

15. M. B. Klein, "Beam coupling in undoped GaAs at $1.06 \mu \mathrm{m}$ using the photorefractive effect," Opt. Lett. 9, 350 (1984).

16. G. Albanese, J. Kumaz, and W. H. Steier, "Investigation of the photorefractive behavior of chrome-doped GaAs by using twobeam coupling," Opt. Lett. 11, 650 (1986).

17. P. Yeh, "Photorefractive two-beam coupling in cubic crystals," J. Opt. Soc. Am. B 4, 1382 (1987).

18. L. J. Cheng, G. Gheen, T. H. Chao, H. K. Liu, A. Partovi, J. Katz, and E. M. Garmire, "Spatial light modulation by beam coupling in GaAs crystals," Opt. Lett. 12, 705 (1987). 
19. J. C. Fabre, J. M. C. Jonathan, and G. Roosen, " $43 \mathrm{~m}$ photorefractive materials in energy transfer experiments," Opt. Commun. 65, 257 (1988)

20. M. Sugie and K. Tada, "Measurements of the linear electrooptic coefficients and analysis of the nonlinear susceptibilities in cubic GaAs and hexagonal CdS," Jpn. J. Appl. Phys. 15, 421 (1976).

21. B. Imbert and J. P. Huignard, Thomson-CSF, B.P. 10, 91401 Orsay, France (personal communication).

22. B. Lambert and Y. Toudic, CNET Lannion, B. 22301 Lannion, France (personal communication).

23. N. V. Kukhtarev, V. B. Markov, S. G. Odulov, M. S. Soskin, and V. L. Vinetskii, "Holographic storage in electro-optic crystals," Ferroelectrics 22, 949 (1979).
24. G. Pauliat, J. M. C. Jonathan, M. Allain, J. C. Launay, and G. Roosen, "Determinations of the photorefractive parameters of $\mathrm{Bi}_{12} \mathrm{GeO}_{20}$ crystals using transient grating analysis," Opt. Commun. 59, 266 (1986); "Optical evidence of a photorefractive effect due to holes in $\mathrm{Bi}_{12} \mathrm{GeO}_{20}$ crystals," Opt. Commun. 61, 321 (1987).

25. B. Clerjaud, "Transition-metal impurities in III-V compounds," J. Phys. C 18, 3615 (1985).

26. D. C. Look, "Model for $\mathrm{Fe}^{2+}$ intracenter-induced photoconductivity in InP:Fe," Phys. Rev. B 20, 10 (1979).

27. P. B. Klein, J. E. Furneaux, and R. L. Henry, "Time-dependent photoluminescence of InP:Fe," Phys. Rev. B 29, 4 (1984).

28. G. C. Valley, "Short-pulse grating formation in photorefractive materials," IEEE J. Quantum Electron. QE-19, 1637 (1983).

\section{J. C. Fabre}

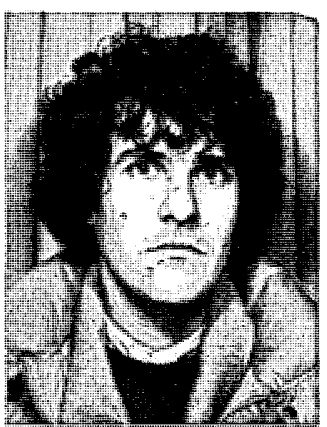

J. C. Fabre graduated from the Université Paris XI, Orsay, France, in 1986. $\mathrm{He}$ is preparing a Ph.D. dissertation on the $\overline{4} 3 \mathrm{~m}$ photorefractive materials and their applications with $\mathrm{G}$. Roosen at the Institut d'Optique Théorique et Appliquée.

\section{J. M. C. Jonathan}

J. M. C. Jonathan was born in Paris in 1950. He received the M.S. degree in physics in 1972 and the Doctorat d'Etat in 1981, both from the Université Pierre et Marie Curie, Paris. Since 1975 he has worked for CNRS at the Institut d'Optique. He described the optically induced anisotropy of silver chloride emulsions and some of its applications to optical data processing. In 1984 he joined the group for nonlinear optics, where he started research on photorefractive materials. In 1986 he spent a year as a research associate with the Department of Electrical Engineering at the University of Southern California, Los Angeles. He is now studying the photorefractive effect in semi-insulators and III-V semiconductor compounds.

\section{G. Roosen}

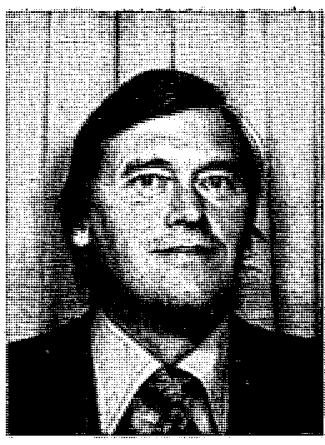

G. Roosen graduated from the Universite Paris XI in 1969. He received the Doctorat-es-Sciences (physics) from this university in 1978. He is Directeur de Recherches, CNRS, and works at the Institut d'Optique Théorique et Appliquée, where he is responsible for the research program on nonlinear-optical phenomena in semi-insulating materials. His initial work was in the field of light radiation pressure, including optical levitation and light-power measurement. He spent a year as a visiting scientist at the IBM Research Laboratory (San Jose, California), where he studied and developed an opto-optical light-steering technique. His current research interests include the basic physics of nonlinear effects in optical materials, short light pulses and their applications to dynamic interconnections, and optical computing. He is a member of the Société Française d'Optique and the Optical Sociey of America. 